\title{
EFEITOS SENSORIAIS DA ADIÇÃO DE EXTRATOS DE DIFERENTES MADEIRAS À CACHAÇA
}

\author{
THIAGO LUCAS DE ABREU-LIMA* \\ AMAZILE BIAGIONI RIBEIRO DE ABREU MAIA** \\ EVELYN DE SOUZA OLIVEIRA***
}

\begin{abstract}
Para avaliar o impacto sensorial da adição de extratos de madeira à cachaça foram produzidos extratos de oito diferentes madeiras: amburana (Amburana cearensis), bálsamo (Miroxylom peruiferum), carvalho brasileiro (Panopsis organensis), carvalho europeu (Quercus alba), ipê amarelo (Tabebuia chrysostrichae), ipê roxo (Tabebuia impetiginosa), jequitibá rosa (Cariniana estrellensis) e louro-canela (Aniba parviflora). Utilizou-se cachaça diluída com água a $8 \% \mathrm{v} / \mathrm{v}$ de etanol como solvente e $30 \mathrm{~g}$ de madeira. Os extratos obtidos foram diluídos 20 vezes com cachaça não-envelhecida e submetidos ao teste de aceitação com escala hedônica não-estruturada. Obtiveram destaque positivo a cachaça com extrato de bálsamo e a cachaça comercial envelhecida em ipê amarelo. As amostras com extratos de louro-canela e jequitibá rosa apresentaram destaque negativo. Tais resultados indicaram a possibilidade da utilização de extratos de madeira no aprimoramento do processo de envelhecimento da cachaça.
\end{abstract}

PALAVRAS-CHAVE: CACHAÇA; AGUARDENTE DE CANA; EXTRATOS DE MADEIRA; ANÁLISE SENSORIAL.

\section{INTRODUÇÃO}

A cachaça é composta por água, etanol e quantidades menores de compostos orgânicos diversos, como alcoóis superiores, ésteres e aldeídos. Os compostos secundários são os responsáveis pelas características sensoriais da bebida.

* $\quad$ Mestre em Ciência de Alimentos, Departamento de Alimentos, Faculdade de Farmácia, Universidade Federal de Minas Gerais (UFMG), Belo Horizonte, MG (e-mail: abreulimatl@gmail.com).

** Doutora em Bioquímica, LABM Pesquisa e Consultoria, Belo Horizonte, MG.

*** Professora, Doutora em Tecnologia de Alimentos, Departamento de Alimentos, Faculdade de Farmácia, UFMG, Belo Horizonte, MG (e-mail: evelyn@farmacia.ufmg.br). 
A produção de cachaça em Minas Gerais foi estimada em 230 milhões de litros anuais, empregando cerca de 240.000 pessoas de forma direta. Aproximadamente 8.500 alambiques são responsáveis por essa produção, com faturamento em torno de 1,5 bilhão de reais ao longo de toda a cadeia produtiva (CARDOSO, 2003; SEBRAE-MG, 2005).

A produção de bebidas alcoólicas pode ser dividida principalmente nas etapas de obtenção e processamento das matérias-primas, fermentação do mosto e destilação do vinho e envelhecimento do destilado para a obtenção do produto acabado (REAZIN, 1981).

O envelhecimento é responsável por melhorias nas características organolépticas da cachaça, tornando seu sabor mais agradável e suave (CARDELLO e FARIA, 1997; CARDELLO e FARIA, 1998; MOSEDALE e PUECH, 1998; DUARTE et al., 2000). Durante o envelhecimento são incorporadas à cachaça substâncias oriundas da madeira. Também ocorrem reações entre os componentes originais do destilado e os componentes extraídos. Desta forma, a bebida envelhecida apresenta menor teor alcoólico e maior concentração de compostos fenólicos e ésteres, características responsáveis pela melhoria em sua aceitação (MOSEDALE e PUECH, 1998). Durante o envelhecimento de bebidas alcoólicas são incorporados ao destilado compostos fenólicos, gerados pela decomposição da lignina, que alteram as propriedades organolépticas da bebida (FURUYA et al., 2000; ISIQUE e FRANCO, 2000). Figuram entre esses compostos a vanilina e os ácidos vanílico e gálico. Já foram encontrados na cachaça kaempeferol, epicatequina, derivados do ácido benzóico e do ácido hidroxicinâmico (ISIQUE e FRANCO, 2000).

O carvalho europeu, tradicionalmente utilizado no envelhecimento de bebidas, tem sido substituído por madeiras nacionais em razão da dificuldade para sua obtenção no Brasil (DIAS, MAIA e NELSON, 1998, DUARTE et al. 2000; YOKOTA et al., 2003).

O tratamento térmico prévio da madeira aumentou o teor de compostos fenólicos em extratos obtidos a partir de amburana, revelando efeito oposto em extratos de bálsamo. Os compostos cujas concentrações diminuíram devido ao tratamento térmico foram a vanilina e o ácido vanílico (CAMPOS et al., 2004).

Diversas pesquisas têm sido desenvolvidas com o objetivo de acelerar, melhorar ou substituir o processo tradicional de envelhecimento de cachaça em barris de madeira. QUEIROZ (1998) estudou a armazenagem 
da cachaça em recipientes de vidro, contendo raspas de madeira isoladamente ou em conjunto com aeração periódica. VALES et al. (2001) adicionaram extratos de madeira à cachaça, produzidos com a utilização de solvente (cachaça retificada, neutralizada e desodorizada com teor alcoólico de $50 \% \mathrm{v} / \mathrm{v}$ ) a quente e em refluxo por 48 horas (VALES et al., 2001). COUTRIM, CHAVES e BRANDÃO (2003) testaram a microxigenação da bebida em processo semelhante ao já utilizado em vinhos. SOUZA e DEL-MASTRO (2004) expuseram a cachaça, acondicionada em recipientes de vidro e madeira à radiação ionizante. FORLIN pesquisou a armazenagem de cachaças em recipientes de polietilenotereftalato (PET) com a adição de extrato de carvalho (FORLIN, 2005).

A análise sensorial, segundo a ABNT (1993), é a "disciplina científica usada para evocar, medir, analisar e interpretar reações das características dos alimentos e materiais como são percebidas pelos sentidos da visão, olfato, gosto, tato e audição". Com técnicas da análise sensorial podem ser descobertas respostas para questões sobre sabor, aroma, aparência, aceitação e atitude do consumidor, impossíveis de serem obtidas por meios instrumentais (STONE e SIDEL, 1993).

A aceitação de aguardente envelhecida em nove diferentes madeiras foi comparada. As amostras envelhecidas em amendoim e pereiro alcançaram valores próximos ao da amostra envelhecida em carvalho. Nessa mesma pesquisa foi fixado em 18 meses o tempo mínimo de envelhecimento necessário para que fossem obtidos bons índices de aceitação, os quais podem variar de acordo com a qualidade da aguardente (BOSCOLO, 1996).

QUEIROZ (1998) pesquisou o envelhecimento forçado da aguardente de cana. Comparou pelo teste de aceitação a amostra testemunha (aguardente de cana não-envelhecida armazenada em garrafão de vidro) e aguardente de cana envelhecida em tonel de carvalho. Os resultados da aceitação para aroma, sabor e impressão global demonstraram que a presença de raspas de carvalho na aguardente e a aeração periódica da mesma podem reduzir o tempo gasto no envelhecimento.

Testes de aceitação, efetuados com 13 amostras comerciais de cachaça não-envelhecida, mostraram que a boa aceitação do aroma pode estar relacionada com a presença de lactonas e alguns terpenos. Já a rejeição do sabor deve-se à presença de fenóis, principalmente 4-vinilfenol e 4vinilguaiacol, conforme correlação entre a aceitação e os resultados de análises químicas (JANZANTTI, 2004). 
Testes de aceitação foram utilizados na análise de amostras maturadas de cachaça com ou sem extrato de carvalho em recipientes de PET, vidro e carvalho. Independente do período de maturação, a adição de 20 , 40 ou $60 \mathrm{~mL}$ de extrato / $\mathrm{L}$ de cachaça aumentou a aceitabilidade das amostras maturadas em recipientes de vidro e PET em relação aos atributos cor, aroma, sabor e impressão global (FORLIN, 2005).

Como o envelhecimento onera o processo de fabricação da cachaça justifica-se a busca de recursos tecnológicos que substituam ou encurtem essa etapa do processo. Um desses recursos seria a incorporação de extratos de madeira à cachaça, logo após a destilação, com o objetivo de encurtar seu tempo de envelhecimento.

Um dos métodos utilizados na extração de compostos vegetais consiste na destilação de solvente na presença do vegetal, de forma que seus componentes sejam extraídos.

Este trabalho teve como objetivo a produção de extratos de madeira por destilação na presença de raspas de madeira e o estudo dos efeitos sensoriais da adição desses extratos à cachaça.

\section{MATERIAL E MÉTODOS}

\subsection{MATERIAL}

\subsubsection{Cachaças}

Foram utilizadas quatro diferentes amostras comerciais de cachaça, todas certificadas pela Associação Mineira de Produtores de Cachaça de Qualidade (AMPAQ). Uma amostra não-envelhecida (NE1) foi usada na obtenção dos extratos. A outra amostra não-envelhecida (NE2) foi empregada na diluição dos extratos para a análise sensorial. As outras duas amostras, envelhecidas em amburana (E1) e ipê amarelo (E2) foram utilizadas na análise sensorial.

\subsubsection{Madeiras}

Para a produção dos extratos foram utilizadas raspas de oito madeiras: amburana (Amburana cearensis), bálsamo (Miroxylom peruiferum), carvalho brasileiro (Panopsis organensis), carvalho europeu (Quercus alba), ipê amarelo (Tabebuia chrysostrichae), ipê roxo (Tabebuia 
impetiginosa), jequitibá rosa (Cariniana estrellensis) e louro-canela (Aniba parviflora). As madeiras nacionais foram cedidas pela Indústria e Comércio Aguardentes Seleta e Boazinha de Salinas, Minas Gerais. O carvalho europeu foi cedido pelo produtor da cachaça "Rumo do Poeta".

\subsection{MÉTODOS}

\subsubsection{Obtenção dos extratos}

Os extratos de madeira foram obtidos pela destilação de aguardente diluída a $8 \%$ de álcool em volume através de raspas de madeira (Figura 1). Cachaça não-envelhecida de boa qualidade (NE1), certificada pela AMPAQ, foi diluída com água destilada até a graduação alcoólica de $8 \%$ v/v para simular a composição do vinho de caldo-de-cana obtido na fermentação de cachaça. Acondicionaram-se $2 \mathrm{~L}$ dessa cachaça diluída no balão de destilação, sendo colocados $30 \mathrm{~g}$ de raspas de madeira na coluna. Conduziu-se a destilação até a obtenção de cerca de $400 \mathrm{~mL}$ de destilado, com concentração de etanol próxima a 42\% v/v. Cada madeira foi submetida ao processo uma vez e a destilação também foi conduzida sem a adição de madeira. Esse destilado e os extratos foram armazenados em garrafas de vidro de $700 \mathrm{~mL}$. Dessa forma, foram obtidos 8 extratos de madeiras e uma amostra de cachaça diluída e redestilada sem madeira (SM).

\section{FIGURA 1 - APARELHO UTILIZADO NA OBTENÇÃO DOS EXTRATOS}

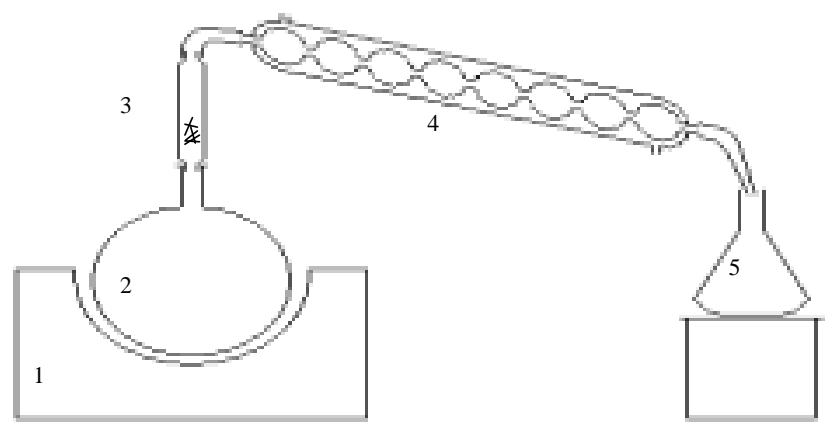

1 = Manta aquecedora com controle de temperatura.

2 = Balão de destilação com capacidade de $3 \mathrm{~L}$.

3 = Coluna confeccionada para a adição das raspas de madeira.

4 = Condensador de bolas de $60 \mathrm{~cm}$ de comprimento.

5 = Frasco para recolhimento da amostra destilada. 


\subsubsection{Análise sensorial - Teste de Aceitação}

Realizou-se a análise de aceitação com quatro escalas hedônicas nãoestruturadas de $9 \mathrm{~cm}$ (STONE e SIDEL, 1993), referentes aos atributos cor, aroma, sabor e impressão global (ancoradas em seus extremos pelos termos "desgostei extremamente" e "gostei extremamente"). Doze amostras foram servidas de forma monádica (porções de $5 \mathrm{~mL}$ ), em taças de vidro incolor (referência S-71080600-02, da marca Nadir Figueiredo), totalizando 12 sessões por julgador.

As 12 amostras analisadas consistiram de oito amostras com extrato de $30 \mathrm{~g}$ de madeira, diluídas 20 vezes com a cachaça NE2 (concentração de extrato $=50 \mathrm{~mL} / \mathrm{L}$ ), a cachaça diluída e destilada na aparelhagem da extração na ausência de madeira (sem madeira - SM), a cachaça utilizada na diluição das amostras (não-envelhecida - NE2) e duas cachaças comerciais envelhecidas em amburana (E1) e ipê amarelo (E2). As amostras comerciais tiveram seu teor alcoólico corrigido para $40 \% \mathrm{v} / \mathrm{v}$ pela adição de água destilada. Cada amostra foi codificada com número aleatório de três dígitos.

Foram recrutados 20 julgadores entre professores e alunos de graduação e de pós-graduação da Faculdade de Farmácia da Universidade Federal de Minas Gerais. A idade dos julgadores variou entre 20 e 32 anos, sendo 5 do sexo masculino e 15 do sexo feminino, os quais foram selecionados por apreciarem o produto em questão.

\subsubsection{Análise estatística dos resultados}

Os resultados do teste de aceitação foram submetidos à análise de variância e comparação de médias pelo teste de Duncan, a 5\% de significância (FERREIRA, 2000).

\section{RESULTADOS E DISCUSSÃO}

Na Tabela 1 encontram-se as notas médias obtidas no teste de aceitação. A partir da análise de variância (quadro ANOVA) foram detectadas diferenças nos quatro atributos analisados, identificadas pelo teste de Duncan (a 5\% de significância). 


\section{TABELA 1 - MÉDIAS DAS NOTAS DE ACEITAÇÃO DAS AMOSTRAS DE CACHAÇA ${ }^{1}$}

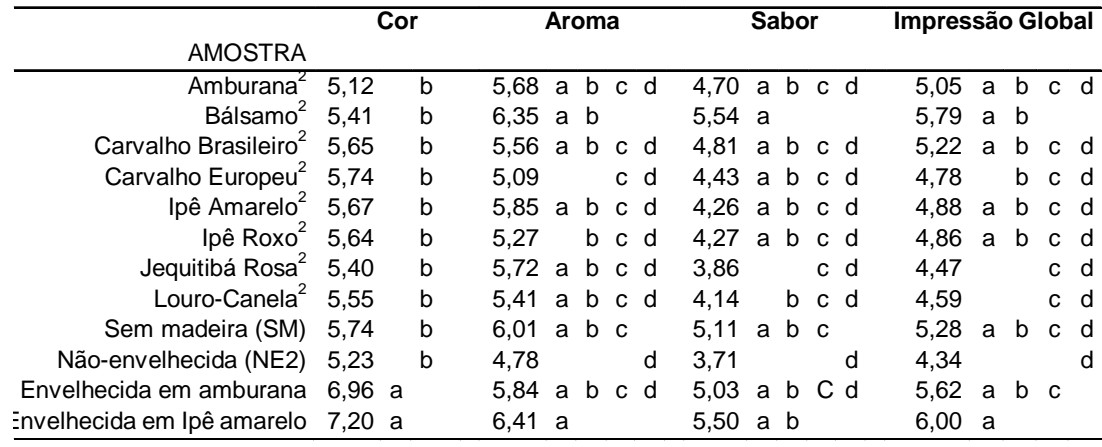

${ }^{1}$ Médias seguidas pela mesma letra na mesma coluna não diferiram entre si pelo teste de Duncan a $5 \%$ de significância. $n=20$ julgadores. Notas variando entre 0 ("desgostei extremamente") e 9 ("gostei extremamente").

${ }^{2}$ Amostra com extrato de $30 \mathrm{~g}$ de madeira, diluídas 20 vezes com a cachaça nãoenvelhecida.

As amostras comerciais envelhecidas em amburana e ipê amarelo evidenciaram melhor aceitação para a cor, não apresentaram diferença entre si e diferiram de todas as outras amostras (que também não diferiram entre si). Tais amostras apresentaram a cor amarelada típica do envelhecimento, destacando-se em relação às demais. Mesmo com aceitação inferior em relação à cor, a média das notas de todas as amostras restantes foi superior a 5,0 (faixa de aprovação).

Em relação ao aroma, a cachaça comercial envelhecida em ipê amarelo diferiu das amostras com extrato de ipê roxo, carvalho europeu e da comercial não-envelhecida. A amostra com extrato de bálsamo, considerada superior, diferiu da amostra com extrato de carvalho europeu e da comercial não-envelhecida. Todas as amostras, exceto a comercial não-envelhecida, obtiveram notas médias superiores a 5,0, (faixa de aprovação) para o aroma. A amostra comercial não-envelhecida obteve média na faixa de indiferença (entre 4,1 e 5,0 ).

As amostras com extrato de bálsamo, comercial envelhecida em ipê amarelo e a sem madeira destacaram-se positivamente em relação ao sabor. As amostras com extrato de louro-canela, jequitibá rosa e a comercial não-envelhecida apresentaram destaque negativo e as amostras restantes obtiveram aceitação intermediária. 
Em relação à impressão global (IG), a amostra comercial envelhecida em ipê amarelo diferiu das amostras com extrato de carvalho europeu, louro-canela, jequitibá rosa e da comercial não-envelhecida. A amostra com extrato de bálsamo, considerada superior, diferiu das amostras com extrato de louro-canela, jequitibá rosa e da comercial não-envelhecida. A amostra comercial envelhecida em amburana diferiu da amostra comercial não-envelhecida. As amostras restantes não diferiram entre si e de nenhuma das outras amostras, apresentando aceitação intermediária para a impressão global. As amostras com extrato de amburana, bálsamo e carvalho brasileiro, a sem madeira e as comerciais envelhecidas (em amburana e ipê amarelo) alcançaram notas médias superiores a 5,0, (faixa de aprovação). A amostra comercial não-envelhecida e as amostras com extrato de carvalho europeu, ipê amarelo, ipê roxo, jequitibá rosa e louro-canela obtiveram média entre 4,1 e 5,0.

É interessante notar que para os atributos aroma, sabor e impressão global a aceitação da amostra com $50 \mathrm{~mL} / \mathrm{L}$ de extrato de bálsamo diferiu da amostra não-envelhecida, composta apenas pela cachaça utilizada na diluição do extrato.

Percebeu-se que algumas amostras destacaram-se pela maior aceitação (envelhecida em ipê amarelo e com extrato de bálsamo), outras pela menor aceitação (não-envelhecida e com extrato de jequitibá rosa, lourocanela e carvalho europeu), obtendo as restantes aceitação intermediária.

A distribuição das freqüências das notas atribuídas pelos julgadores (Figuras 2 a 4) confirmou as diferenças entre as amostras.

Verificou-se maior índice de rejeição para o aroma da amostra nãoenvelhecida (40\%). Essa amostra e a com extrato de jequitibá rosa evidenciaram também os maiores índices de rejeição para sabor e impressão global, respectivamente 55\% e $45 \%$.

O maior índice de aprovação para o aroma (85\%) foi obtido pela amostra comercial envelhecida em ipê amarelo, seguida pela amostra com extrato de bálsamo (75\% de aprovação). A amostra com extrato de bálsamo alcançou os maiores índices de aprovação para o sabor e impressão global (respectivamente $65 \%$ e $70 \%$ ), seguida em ambos os casos pela amostra comercial envelhecida em ipê amarelo (60\% e 65\%). Apenas as amostras com extrato de bálsamo e a comercial envelhecida em ipê amarelo não apresentaram rejeição superior a $25 \%$ em nenhum dos atributos e aprovação de no mínimo 50\% em todos eles. Diante de tais 
dados confirmou-se a superioridade dessas duas amostras perante as demais.

FIGURA 2 - REPRESENTAÇÃO GRÁFICA DO PERCENTUAL DE REJEIÇÃO DAS AMOSTRAS (AROMA, SABOR E IMPRESSÃO GLOBAL)

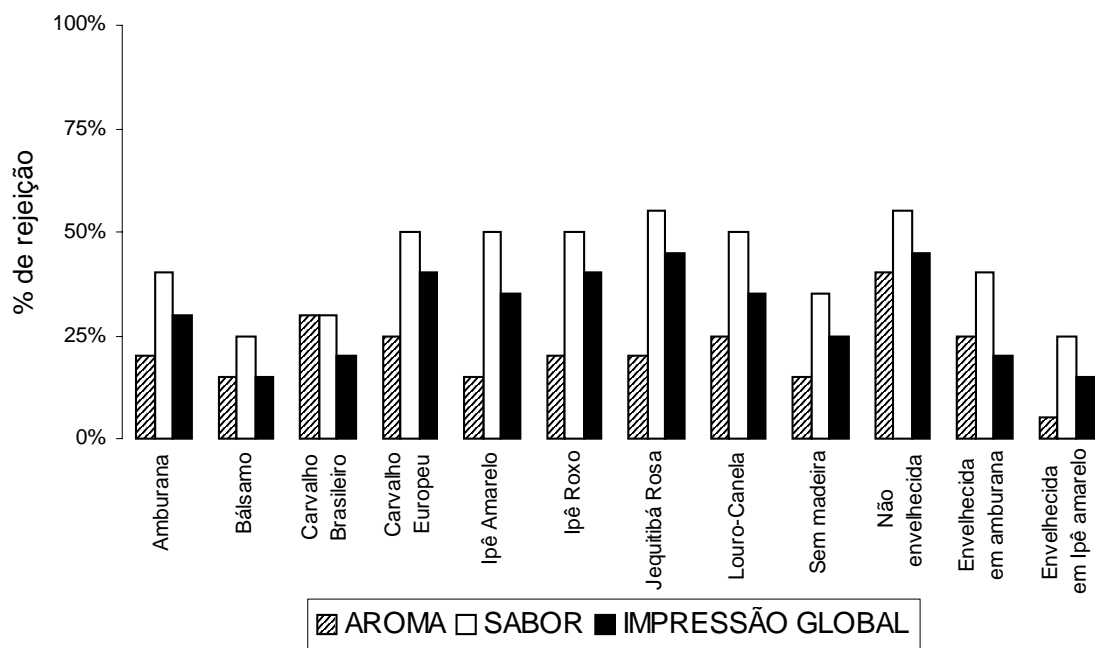

FIGURA 3 - REPRESENTAÇÃO GRÁFICA DO PERCENTUAL DE INDIFERENÇA PARA OS ATRIBUTOS AROMA, SABOR E IMPRESSÃO GLOBAL DAS AMOSTRAS

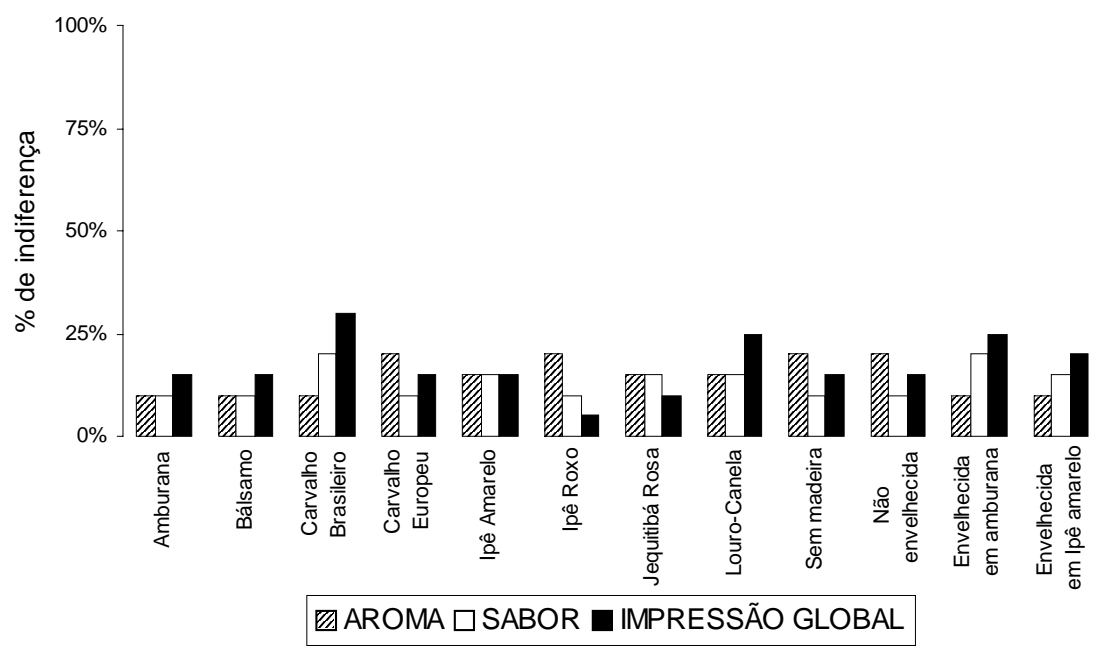


O único atributo com rejeição igual ou superior a $50 \%$ foi o sabor (amostra não-envelhecida e amostras com extrato de carvalho europeu, ipê amarelo, ipê roxo, jequitibá rosa e louro-canela). As amostras menos aceitas conforme o teste de Duncan foram a não-envelhecida e as com extrato de carvalho europeu, jequitibá rosa e louro-canela. As outras duas, com extrato de ipê roxo e ipê amarelo, diferenciam-se daquelas quatro por apresentarem índice de aceitação em relação à impressão global de no mínimo 50\% (Figura 4).

\section{FIGURA 4 - REPRESENTAÇÃO GRÁFICA DO PERCENTUAL DE APROVAÇÃO DAS AMOSTRAS (AROMA, SABOR E IMPRESSÃO GLOBAL)}

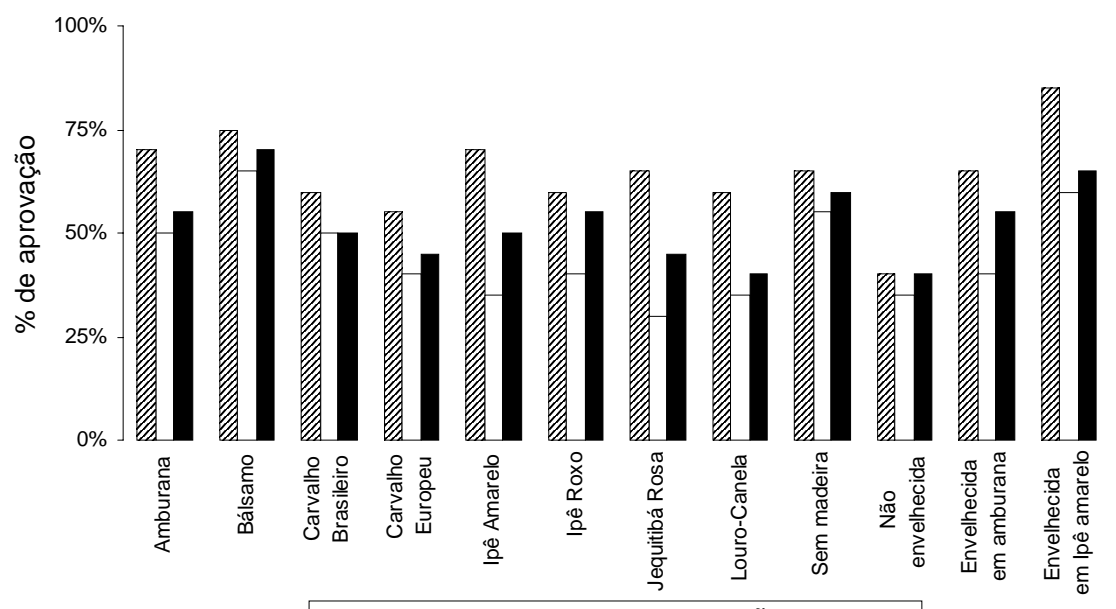

図 AROMA $\square$ SABOR $\square$ IMPRESSÃO GLOBAL

A análise da distribuição das freqüências das notas complementou o resultado obtido com o teste de Duncan, evidenciando maior aceitação para a amostra comercial envelhecida em ipê amarelo e para a com extrato de bálsamo. Tal resultado surpreende pelo fato do ipê amarelo não ser tradicionalmente utilizado no envelhecimento de bebidas destiladas.

É interessante observar que a amostra com extrato de amburana e a comercial envelhecida em amburana tiveram o mesmo índice de rejeição para o sabor (40\%) e o mesmo índice de aprovação para impressão 
global (55\%). Já a amostra com extrato obteve maiores índices de aprovação para aroma e sabor que a amostra envelhecida.

O destaque positivo do bálsamo e negativo do louro já tinha sido observado por FARIA et al. (2003) em amostras de cachaça armazenadas durante 6 meses em recipientes de diferentes madeiras (carvalho, pau d'arco, amendoim, pereiro, jatobá, bálsamo, amarelo, pau d'óleo e louro).

A vanilina, o segundo composto odorífero mais potente e com a segunda maior concentração, foi detectada em amostra comercial de cachaça de alta qualidade pela técnica CG-olfatometria, sendo descrita como "muito doce" (JANZANTTI, 2004). A vanilina destaca-se em cachaças envelhecidas em bálsamo e o ácido vanílico em cachaças envelhecidas em amburana, ipê ou jequitibá (DIAS, 1997). O aquecimento do bálsamo a $200^{\circ} \mathrm{C}$ por $30 \mathrm{~min}$ (CAMPOS et al., 2004) e a 180 $\mathrm{C}$ por $2 \mathrm{~h}$ (DIAS, 1997), antes da obtenção de extratos, acarretou decréscimo na concentração de vanilina, sugerindo que esse seja decomposto ou volatilizado com a temperatura. Pode-se supor que a vanilina presente no bálsamo, seja incorporada no extrato produzido por destilação através de camada de raspas de madeira, o que explicaria a boa aceitação da amostra com extrato de bálsamo obtida no presente trabalho.

\section{CONCLUSÃO}

Os extratos de amburana, bálsamo, carvalho brasileiro, carvalho europeu, ipê amarelo, ipê roxo, jequitibá rosa e louro-canela obtidos por destilação apresentaram aromas característicos de bebidas alcoólicas envelhecidas.

Não era esperado que a adição de extrato por si só fosse capaz de produzir melhorias definitivas no sabor da cachaça, pois não há tempo para que ocorram todas as reações envolvidas na maturação de bebidas, especialmente a oxidação dos componentes secundários e as reações de esterificação. A adição de tais extratos à cachaça alterou suas características sensoriais de forma perceptível. A amostra de cachaça não-envelhecida com a adição de $50 \mathrm{~mL} / \mathrm{L}$ de extrato de bálsamo destacou-se em relação ao aroma, sabor e impressão global. Essa amostra foi considerada superior à cachaça com extrato de carvalho europeu em relação ao aroma e às cachaças com extrato de jequitibá rosa e louro-canela quanto ao sabor e impressão global. A superioridade na aceitação de tais atributos pode ter sido ocasionada pela vanilina, já que sua presença foi evidenciada em extratos de bálsamo e em cachaças envelhecidas em bálsamo. A cachaça envelhecida em ipê amarelo 
destacou-se de modo surpreendente no teste de aceitação, já que o ipê amarelo não é tradicionalmente utilizado no envelhecimento de bebidas destiladas.

Os resultados encontrados representam o primeiro passo em direção à utilização de extratos de madeira obtidos por destilação para melhoria das características sensoriais da cachaça. Sugere-se a realização de trabalhos sobre a caracterização química dos extratos, correlacionandoos com os extratos obtidos, com outras técnicas e com amostras de cachaça, bem como o estudo da adição de extratos associados com outras técnicas, tais como microxigenação, aeração, irradiação e adição de açúcar.

\section{Abstract}

\section{SENSORIAL EFFECTS OF THE ADDITION OF DIFFERENT WOOD EXTRACTS} TO SUGAR CANE SPIRIT

To evaluate the sensorial impact of the addition of wood extracts to sugar cane spirits, extracts were produced from eight different woods: amburana (Amburana cearensis), balm (Miroxylom peruiferum), Brazilian oak (Panopsis organensis), European oak (Quercus alba), yellow ipê (Tabebuia chrysostrichae), purple ipê (Tabebuia impetiginosa), pink jequitibá (Cariniana estrellensis) and louro-canela (Aniba parviflora). Used sugar cane spirit was diluted with $8 \% \mathrm{v} / \mathrm{v}$ of ethanol as solvent and $30 \mathrm{~g}$ of wood. The obtained extracts were diluted 20 times with a non-aged sugar cane spirit and submitted to an affective testing by non structured hedonic scale. The spirits with balm extract and the commercial sample aged in yellow ipê showed higher scores. The samples with louro-canela and pink jequitibá extracts showed the lowest scores. These results show the possibility of the utilization of wood extracts to improve the beverage ageing process.

KEY-WORDS: SUGAR CANE SPIRIT; WOOD EXTRACTS; SENSORY ANALYSIS.

\section{REFERÊNCIAS}

1 ABNT. Associação Brasileira de Normas Técnicas. Análise sensorial de alimentos e bebidas - NBR 12806. São Paulo, 1993.

2 BOSCOLO, M.. Estudo sobre envelhecimento de aguardente de cana-deaçúcar. São Carlos, 1996. 83 p. Dissertação (Mestrado em Ciências: Química Analítica), Instituto de Química, Universidade de São Paulo.

3 CAMPOS, J.O.S.; AQUINO, F.W.B.; NASCIMENTO, R.F.; COSTA, J.G.M.; KEUKELEIRE, D.D.; CASIMIRO, A.R.S. Influence and effect of thermal treatment in elaboration of regional wood extracts for cachaça. Journal of Food Composition and Analysis, v. 17, p. 179-185, 2004. 
aguardente de cana durante o envelhecimento em tonel de carvalho (Quercus alba L.). Boletim do CEPPA, Curitiba, v. 15, n. 2, p. 87-100, 1997.

5 CARDELLO, H.M.A.B.; FARIA, J.B. Análise descritiva quantitativa da aguardente de cana durante o envelhecimento em tonel de carvalho (Quercus alba L.). Ciênc. Tecnol. Aliment., v. 18, p. 169-175, 1998.

6 CARDOSO, M.G. Controle de qualidade de cachaça. In: EXPOCACHAÇA2003, 2003, Belo Horizonte. Anais ... Belo Horizonte: Rede Mineira de Tecnologia da Cachaça, 2003. p. 14-15.

7 COUTRIM, M.X.; CHAVES, J.B.; BRANDÃO, R.L. Utilização da microxigenação como alternativa ao processo de envelhecimento da cachaça de alambique. In: EXPOCACHAÇA2003, 2003, Belo Horizonte. Anais ... Belo Horizonte: Rede Mineira de Tecnologia da Cachaça, 2003. p. 24.

8 DIAS, S.; MAIA, A.; NELSON, D. Efeito de diferentes madeiras sobre a composição da aguardente de cana envelhecida. Ciênc. Tecnol. Aliment., v. 18, p. 169175, 1998.

9 DIAS, S.M.B.C. Efeito de diferentes tipos de madeira sobre a composição química da aguardente de cana envelhecida. Belo Horizonte, 1997. 109 p. Dissertação (Mestrado em Ciência de Alimentos), Faculdade de Farmácia, Universidade Federal de Minas Gerais.

10 DUARTE, R.M.; NOBUKUNI, S.L.; MUTTON, M.A.; MUTTON, M.J.R. Analysis of sugar cane spirit submitted to the process of aging in different wood. In: BRAZILIAN MEETING ON CHEMISTRY OF FOOD AND BEVERAGES, 3, 2000, São Carlos. Book of Abstracts ... São Carlos: Instituto de Química de São Carlos da Universidade de São Paulo, 2000. p. 1.

11 FARIA, J.B.; CARDELLO, H.M.A.B.; BOSCOLO, M.; ISIQUE, W.D.; ODELLO, L.; FRANCO, D.W. Evaluation of Brazilian woods as an alternative to oak for cachaças aging. Eur. Food Res. Technol., n. 218, p. 83-87, 2003.

12 FERREIRA, V.L.P. (Coord.) Análise sensorial: testes discriminativos e afetivos. São Paulo: Sociedade Brasileira de Ciência e Tecnologia de Alimentos, 2000. $127 \mathrm{p}$.

13 FORLIN, F.J.. Maturação de cachaça em recipientes de poliéster com extrato de madeira de carvalho. Campinas, 2005. 174 p. Tese (Doutorado em Tecnologia de Alimentos), Faculdade de Engenharia de Alimentos, Universidade Estadual de Campinas.

14 FURUYA, S.M.B.; BENASSI NETO, M.; LIMA NETO, B.S.; FRANCO, D.W. Analysis of phenolic compounds in alcoholic beverages. In: BRAZILIAN MEETING ON CHEMISTRY OF FOOD AND BEVERAGES, 3, 2000, São Carlos. Book of Abstracts ... São Carlos: Instituto de Química de São Carlos da Universidade de São Paulo, 2000. p. 38. 
15 ISIQUE, W.D.; FRANCO, D.W. Phenolic compounds present in sugar cane spirits. In: BRAZILIAN MEETING ON CHEMISTRY OF FOOD AND BEVERAGES, 3, 2000, São Carlos. Book of Abstracts ... São Carlos: Instituto de Química de São Carlos da Universidade de São Paulo, 2000. p. 63.

16 JANZANTTI, N.S. Compostos voláteis e qualidade de sabor da cachaça. Campinas, 2004. 179 p. Tese (Doutorado em Ciência de Alimentos), Faculdade de Engenharia de Alimentos, Universidade Estadual de Campinas.

17 MOSEDALE, J.R.; PUECH, J.L. Wood maturation of distilled beverages. Trends in Food Science \& Technology, v. 9, p. 95-101, 1998.

18 QUEIROZ, E.L.M.T. Envelhecimento forçado da aguardente de cana: estudo comparativo com o envelhecimento tradicional em carvalho (Quercus $s p$ ). Araraquara, 1998. 83 p. Dissertação (Mestrado em Alimentos e Nutrição), Faculdade de Ciências Farmacêuticas, Universidade Estadual Paulista "Júlio de Mesquita Filho"

19 REAZIN, G.H. Chemical mechanisms of whiskey maturation. Am. J. Enol. Vitic., v. 32 , p. $283-289,1981$.

20 SEBRAE-MG. Serviço de Apoio às Micro e Pequenas Empresas de Minas Gerais. Diagnóstico da cachaça de Minas Gerais. Belo Horizonte: 2001. 241 p. Disponível em: <http://www.sebraemg.com.br/agronegocios/Cachaca/ Cacha\%E7a\%20MG.pdf> Acesso em: 3 mar. 2005.

21 SOUZA, M.D.C.A.; DEL-MASTRO, N.L.. Efeito da radiação gama na aguardente de cana-de-açúcar. Brazilian Journal of Food Technology, v. 7, n. 1, p. 9-15, 2004.

22 STONE, H.; SIDEL, J.L. Sensory evaluation practices. $2^{\text {nd }}$ ed. San Diego: Academic Press, 1993. 338 p.

23 VALES, R.M.S.; PINHEIRO, S.H.M.; CAMPOS, J.O.S.; FILHO, M.M.S.; CASIMIRO, A.R.S. Efeito dos extratos aromáticos de madeiras sobre cachaças. Engarrafador Moderno, n. 4, p. 40-47, 2001.

24 YOKOTA, S.R.C.; CHAVES, J.B.P.; COSTA, M.S.; MINIM, V.P.R.; SILVA, P.H.A. Mapa de preferência interno de cachaças envelhecidas em barril de carvalho ou tonéis de madeiras brasileiras. In: EXPOCACHAÇA2003, 2003, Belo Horizonte. Anais ... Belo Horizonte: Rede Mineira de Tecnologia da Cachaça, 2003. p. 27. 